\title{
A description of premature and ex-premature infants admitted to the paediatric intensive care unit in the first six months of life
}

\author{
G Mathew, ${ }^{1,2}$ MB BCh, DCH (SA), FC Paed (SA); B Rossouw, ${ }^{1,2}$ MB ChB, DTM, MSc, MMed (Paed), Cert Crit Care (Paed); \\ B M Morrow, ${ }^{1}$ PhD, PG Dip Health Res Ethics, PG Dip Palliative Med (Paed), BSc Physiotherapy \\ ${ }^{1}$ Department of Paediatrics and Child Health, Faculty of Health Sciences, University of Cape Town, South Africa \\ ${ }^{2}$ Paediatric Intensive Care Unit, Red Cross War Memorial Children's Hospital, Cape Town, South Africa
}

Corresponding author: B M Morrow (brenda.morrow@uct.ac.za)

\begin{abstract}
Background. Prematurity is a major risk factor for paediatric morbidity and mortality. Rehospitalisation with paediatric intensive care unit (PICU) admission constitutes significant morbidity; however, the extent of this problem in South Africa (SA) is not known.

Objective. To describe the outcomes, clinical course and characteristics of premature and ex-premature infants admitted to a SA PICU, and to determine predictors of mortality.

Methods. This prospective observational study analysed unplanned PICU admissions of infants in the first six months of life, over a six-month period. The primary outcome was mortality. Data were analysed using standard descriptive and inferential statistics.

Results. Included in the study were 29 infants (65\% male; median (interquartile range) birthweight and gestational age 1715 (1 130 - 2340$) \mathrm{g}$ and $32(29$ - 34) weeks, respectively) in 33 admissions. Five (17.2\%) infants died in the PICU. Apnoea (39.4\%), respiratory failure (24.2\%) and shock (24.2\%) were the most common reasons for PICU admission, secondary to pneumonia (33.3\%), sepsis (27.3\%) and meningitis (12.1\%); $72.4 \%$ of infants were mechanically ventilated; and $48.3 \%$ received blood transfusions. Higher revised paediatric index of mortality score ( $p=0.03)$, inotrope use $(p<0.0001)$, longer duration of mechanical ventilation $(p=0.03)$, and cardiac arrest in the PICU $(p<0.0001)$ were associated with mortality on univariate analysis with no independent predictors of mortality.

Conclusion. Infections leading to apnoea, respiratory failure and shock are common indications for PICU readmission in premature infants. Mechanical ventilation and blood transfusion were frequently required.
\end{abstract}

S Afr J Child Health 2020;14(4):185-191. https://doi.org/10.7196/SAJCH.2020.v1414.1692

Prematurity is a major risk factor for morbidity and mortality, with preterm birth complications being the leading cause of death in children $<5$ years. $^{[1]}$ In the Cape Town Metro West geographical service area of Western Cape Province, South Africa (SA), prematurity is the third leading cause of death; accounting for $9.3 \%$ of all under- 5 deaths in the region. ${ }^{[2]}$ There have been dramatic advancements in the knowledge and care of premature infants, with associated improvements in survival, even in cases which would previously have been considered futile. These interventions include the use of incubators, surfactant and continuous positive airway pressure.$^{[3]}$ However, these premature infants may survive with significant morbidity, ${ }^{[4]}$ with associated increased demands on the healthcare system and family.

Studies conducted in well-resourced countries have identified premature infants as being at high risk of requiring readmission to hospital, ${ }^{[5,6]}$ with those readmitted to the neonatal or paediatric intensive care unit (PICU) being at increased risk of poor clinical outcomes (prolonged PICU stay and mortality) compared with the general PICU population..$^{[7]}$ Male sex, ${ }^{[5,8-12]}$ chronic lung disease/ bronchopulmonary dysplasia, ${ }^{[8,11,13]}$ lower gestational age (GA) at birth and lower birthweight $(\mathrm{BW})^{[5,9,13,14]}$ have been associated with an increased risk of rehospitalisation, particularly a BW $<1000$ g. ${ }^{[5,13]}$ Kuint et al. ${ }^{[15]}$ found that the presence of any morbidity associated with prematurity increased the rate of rehospitalisation in very low-birthweight infants (VLBW) until 18 years of age in Israel. Breastfeeding has been identified as a protective factor ${ }^{[8,16]}$ reducing the likelihood of rehospitalisation. Acute respiratory illnesses, particularly lower respiratory tract infections, have consistently been reported to be a common reason for readmission into ICU. ${ }^{[17]}$

Across 16 centres in the USA, ${ }^{[7]}$ premature infants had a high rate of rehospitalisations (18.7\%), especially during the first year of life, and required prolonged ICU stays. There are limited data on the characteristics, outcomes and impact on the healthcare system of infants requiring readmission to PICU in low- and middleincome countries, with no studies from sub-Saharan Africa. A study from India found that infections (sepsis, pneumonia and acute gastroenteritis) lead to rehospitalisation in the majority of cases in extremely low-birthweight (ELBW) infants in the first year of life. ${ }^{[18]}$ Forty-four percent of their cohort of ELBW infants were rehospitalised and $11 \%$ of these died within the first year of life. It is particularly important to identify infants at increased risk of poor clinical outcomes in resource-constrained environments, as they may require closer surveillance and support.

The present study aims to describe the incidence, demographics, clinical course and outcomes of premature infants admitted to a South African PICU, following initial hospital discharge, within the first 6 months of life.

\section{Methods}

\section{Study design and participants}

This was a prospective observational study of all premature and ex-premature infants below the age of 6 months, admitted to the PICU at Red Cross War Memorial Children's Hospital (RCWMCH), Cape Town, SA, from 1 May 2016 to 31 October 2016. All planned 
admissions, such as those admitted following elective surgery, were excluded from the study. The Human Research Ethics Committee (HREC) of the University of Cape Town granted ethical approval for this study (HREC ref. no. 103/2016) and informed consent was taken from participants' parents or legal guardians.

\section{Study setting}

RCWMCH is the only paediatric tertiary level (L3) hospital within the Cape Town Metro West geographical service area in Western Cape, offering a wide scope of subspecialty services. The PICU at $\mathrm{RCWMCH}$ is a 22-bed unit providing the only intensive care unit (ICU) for paediatric admissions within the public health sector in the region. Neonatal services within this district are offered by one L3 hospital, several secondary level (L2) hospitals and district level (L1) hospitals, and multiple maternity obstetric units. Usually, once an infant is discharged from the neonatal services, any rehospitalisations required would be to a paediatric facility. During the study period, PICU staffing and admission criteria remained fairly stable.

\section{Data collection}

The PICU was screened daily to identify eligible participants. Birthweight, gestational age at birth (as determined by the attending clinician), gender, mode of delivery, and date of birth were recorded for each participant from their medical records, and corrected and chronological ages were calculated for each child. Premature and underweight infants were categorised according to the World Health Organization (WHO) criteria ${ }^{[19]}$ Children born before 28 weeks' gestation were classified as extremely preterm, those born between 28 and $<32$ weeks' gestation as very preterm, and those born between 32 and $<37$ weeks as moderate to late preterm. Data regarding initial hospital length of stay following birth, morbidities, NICU discharge weight, immunisation status and human immunodeficiency virus (HIV) status were extracted from Road to Health booklets.

HIV status was classified as uninfected, exposed but uninfected, infected not on treatment, and infected on treatment.

Immunisation status was based on the expected immunisations that each child should have received at the time of admission, based on the South African Extended Programme on Immunisation (EPI).

The weight of the infant on the day of admission to the PICU was used to assess the child's growth since discharge from the neonatal unit. The nutritional status of each child was determined using weight-for-age (WAZ) and weight-for-height/length (WHZ) $z$-scores after correction for prematurity, calculated using the zanthro function of Stata/IC 13.0 statistical software (StataCorp., USA). The WHO 2007 UK term and preterm growth charts were used for reference (Birth: British 1990 Growth Reference, reanalysed 2009; Postnatal: WHO Child Growth Standards; 4 - 20 years: British 1990 Growth Reference). A child was assessed as growing slowly if they had gained weight since the time of discharge from the neonatal unit but had not followed the trend according to the previously mentioned growth charts.

The following data were recorded regarding the PICU admission: initial reason for admission; Paediatric Index of Mortality score (PIM2); discharge diagnosis; mode of feeding (breastmilk, formula feeds or mixed feeding); length of stay; respiratory support; blood products received; unexpected interventions such as surgery or dialysis; and laboratory results (haemoglobin $(\mathrm{Hb})$, microbiological and viral studies). The PIM2 score uses various physiological variables to determine mortality risk assessment at PICU admission. ${ }^{[20]}$ It has been validated for use in the PICU at RCWMCH. ${ }^{[21]}$

\section{Data analysis and main outcome measures}

Data were tested for normality using the Shapiro-Wilk W-test. Descriptive data are presented as $n$ (\%) for categorical variables and either median (interquartile range (IQR)) or mean (standard deviation (SD)) according to distribution for continuous variables. Inferential statistics were conducted using $\chi^{2}$ tests (or Yates-corrected $\chi^{2}$ as appropriate) for categorical variables and continuous data were compared using Mann-Whitney $\mathrm{U}$ - or $t$-tests for independent variables. The primary outcome measure was mortality. Data identified as being significantly associated with mortality on univariate analysis were entered into a backward stepwise binary logistic regression model to determine any independent predictors. A $p$-value $<0.05$ was considered significant for all tests. Statistica 13 (StatSoft Inc., USA) was used for statistical analysis.

\section{Results}

During the 6-month study period, there were 584 admissions to the PICU, of which 33 (5.7\%) were unplanned admissions of 29 prematurely born infants (male, $n=19 ; 65.5 \%$ ). Within the same period, there were a total of 43 deaths in the PICU, 5 (11.6\%) of whom were patients included in the present study (Table 1).

\section{Patient characteristics and outcomes}

Characteristics of the 29 included infants are presented in Table 1. One (3.4\%) child had a GA of 24 weeks; 11 (37.9\%) were very preterm and $17(58.6 \%)$ were moderate to late preterm infants. Eight $(27.6 \%)$ infants were discharged from the neonatal unit with a known morbidity, the most common of these being a late infection (10.3\%) during the birth hospitalisation. One of the infants had a grade 3 - 4 intraventricular haemorrhage, and another had necrotising enterocolitis during the neonatal admission. More than half of the infants $(51.7 \%)$ admitted to the PICU had a neonatal length of stay exceeding 4 weeks.

Of the 29 infants requiring readmission to PICU, 9 (31\%) were noted to have a WAZ or WHZ below -2 at the time of readmission or had lost weight since discharge from the neonatal unit. These children were classified as malnourished (Table 1). All p-values (Tables 1 and 3) refer to the comparison between patients who died and those who survived.

The most common reason for PICU admission was apnoea (Table 2). Infants with pneumonia who had no evidence of nonpulmonary organ dysfunction during PICU admission were given a discharge diagnosis of pneumonia. Any child with multi-organ dysfunction secondary to pulmonary or non-pulmonary infection during the admission to the PICU were classified as having sepsis on discharge. Infections were the most common discharge diagnoses (75.8\%) (Table 2); $42.4 \%$ of these infants were admitted with respiratory illness, including those with pneumonia, bronchiolitis and upper airway obstruction.

The vast majority of admissions secondary to infective causes had no identified microbial diagnosis; one infant was found to have a growth of Group B streptococcus on blood culture. One child was treated as a presumed Bordetella pertussis infection, despite having received 3 doses of the EPI-funded B. pertussis vaccination. Respiratory syncytial virus (RSV) was isolated in 4 (13.8\%) patients, 3 of whom were diagnosed with respiratory tract infections. Two of these patients were admitted during the RSV season and none of the 29 infants received palivizumab.

Twenty-four of the 33 admissions (72.7\%) were intubated and mechanically ventilated during the course of the PICU stay. Table 3 presents total duration of PICU stay and mechanical ventilation for the included infants. 


\begin{tabular}{|c|c|c|c|c|}
\hline Variable & All $(N=29), n(\%)^{*}$ & $\operatorname{Died}(n=5), n(\%) *$ & Survived $(n=24), n(\%)^{*}$ & $p$-value \\
\hline Male gender & $19(65.5)$ & $4(80.0)$ & $15(62.5)$ & 0.5 \\
\hline Delivery by caesarean section & $8(27.6)$ & $1(20.0)$ & $7(29.2)$ & 0.7 \\
\hline $\mathrm{BW}(\mathrm{g})$, median (IQR) & $1715(1130-2340)$ & $1800(1180-2410)$ & $1667.5(1115-2320)$ & 0.8 \\
\hline GA (weeks), median (IQR) & $32(29-34)$ & $32(28-34)$ & $32.5(29-34)$ & 0.9 \\
\hline PIM2 score (\%), median (IQR) & $4.13(1.36-7.56)$ & $13.17(7.56-62.23)$ & $3.8(1.08-5.53)$ & 0.03 \\
\hline \multicolumn{5}{|l|}{ Length of stay in neonatal unit } \\
\hline None & $4(13.8)$ & $2(40.0)$ & $2(8.3)$ & 0.4 \\
\hline$<4$ days & $2(6.9)$ & 0 & $2(8.3)$ & \\
\hline $4-<7$ days & $2(6.9)$ & 0 & $2(8.3)$ & \\
\hline 1 - 2 weeks & $5(17.2)$ & $1(20.0)$ & $4(16.7)$ & \\
\hline 2 - 4 weeks & $1(3.4)$ & 0 & $1(4.2)$ & \\
\hline$>4$ weeks & $15(51.7)$ & $2(40.0)$ & $13(43.2)$ & \\
\hline Presence of morbidities & $8(27.6)$ & $2(40.0)$ & $6(25.0)$ & 0.4 \\
\hline \multicolumn{5}{|l|}{ Corrected age on PICU admission } \\
\hline $35-<37$ weeks & $7(24.1)$ & $1(20.0)$ & $6(25.0)$ & 0.8 \\
\hline $37-<40$ weeks & $7(24.1)$ & $2(40.0)$ & $5(20.8)$ & \\
\hline 40 weeks $-<1$ month & $6(20.7)$ & $1(20.0)$ & $5(20.8)$ & \\
\hline 1 to $<3$ months & $5(17.2)$ & $1(20.0)$ & $4(16.7)$ & \\
\hline$\geq 3$ months & $4(13.8)$ & 0 & $4(16.7)$ & \\
\hline \multicolumn{5}{|c|}{ Actual age on PICU admission, months } \\
\hline$<1$ & $8(27.6)$ & $2(40.0)$ & $6(25.0)$ & 0.7 \\
\hline $1-<2$ & $7(24.1)$ & $1(20.0)$ & $6(25.0)$ & \\
\hline $2-<4$ & $10(34.5)$ & $2(40.0)$ & $8(33.3)$ & \\
\hline $4-6$ & $4(13.8)$ & 0 & $4(16.7)$ & \\
\hline \multicolumn{5}{|l|}{ Admission weight, $\mathrm{kg}$} \\
\hline$<2$ & $6(20.7)$ & 0 & $6(25.0)$ & 0.4 \\
\hline $2-<4$ & $19(65.5)$ & $5(100)$ & $14(58.3)$ & \\
\hline $4-<6$ & $3(10.3)$ & 0 & $3(12.5)$ & \\
\hline$\geq 6$ & $1(3.4)$ & 0 & $1(4.2)$ & \\
\hline \multicolumn{5}{|l|}{ Growth since discharge } \\
\hline Growing well & $14(48.3)$ & $3(60.0)$ & $11(45.8)$ & 0.5 \\
\hline Growing slowly & $6(20.7)$ & 0 & $6(25.0)$ & \\
\hline Malnourished or weight loss & $9(31.0)$ & $2(40.0)$ & $7(13.4)$ & \\
\hline \multicolumn{5}{|l|}{ Time since neonatal unit discharge } \\
\hline$<1$ week & $2(6.9)$ & 0 & $2(8.3)$ & 0.1 \\
\hline 1 - 2 weeks & $3(10.3)$ & 0 & $3(12.5)$ & \\
\hline$>2$ weeks - 1 month & $8(27.6)$ & $4(80.0)$ & $4(16.7)$ & \\
\hline$>1-<2$ months & $10(34.5)$ & $1(20.0)$ & $9(37.5)$ & \\
\hline$\geq 2$ months & $6(20.7)$ & 0 & $6(25.0)$ & \\
\hline Immunisations not up to date & $11(37.9)$ & 0 & $11(45.8)$ & 0.1 \\
\hline \multicolumn{5}{|l|}{ Feeding } \\
\hline Breast & $21(72.4)$ & $4(80.0)$ & $17(70.8)$ & 0.7 \\
\hline Formula & $5(17.2)$ & $1(20.0)$ & $4(16.7)$ & \\
\hline Mixed & $3(10.3)$ & 0 & $3(12.5)$ & \\
\hline \multicolumn{5}{|l|}{ HIV status } \\
\hline HIV-positive & 0 & 0 & 0 & 0.3 \\
\hline HIV-exposed, but negative & $4(13.8)$ & 0 & $4(16.7)$ & \\
\hline HIV-unexposed & $25(86.2)$ & $5(100)$ & $20(59.0)$ & \\
\hline
\end{tabular}

One child required emergency dialysis and 7 (24.1\%) infants underwent surgery during their PICU admission: laparotomy $(n=3)$; tracheostomy $(n=2)$; patent ductus arteriosus ligation $(n=1)$ and removal of ventriculo-peritoneal shunt $(n=1)$. One of these children, who underwent laparotomy for an incarcerated hernia, died while the remainder survived. The one child who required dialysis died.

Infants who received blood transfusions had lower median (IQR) birthweight (1 170 
(1 000 - 1715$)$ g v. 2300 (1 620 - 2 410) g; $p=0.01)$ and younger median (IQR) gestational age $(29.5(28$ - 32) weeks v. 33 (31 36) weeks; $p=0.02$ ) compared with those who did not receive transfusions. The median haemoglobin for which a transfusion was administered was $8.5 \mathrm{~g} / \mathrm{dL}$.

Five infants (17\%) were successfully resuscitated following at least one cardiac arrest in the PICU (Table 4). However, all of these infants eventually died in the PICU.

\section{Outcomes}

Five (17.2\%) infants died in the PICU. The standardised mortality ratio (actual/mean predicted mortality, SMR) was 1.39 .

Higher PIM2 score on admission, a longer period of mechanical ventilation, receipt of inotropes, and cardiac arrest were associated with PICU mortality on univariate analysis (Table 3). However, none of these associations was found to be an independent predictor of

Table 2. Reason for admission and discharge diagnosis ( $N=33$ admissions)

\begin{tabular}{ll}
\hline Reason for admission $(N=33), n(\%)$ & \\
\hline Apnoea & $13(39.4)$ \\
Respiratory failure & $8(24.2)$ \\
Septic shock & $5(15.2)$ \\
Hypovolaemic shock & $3(9.1)$ \\
Cardiac arrest & $2(6.1)$ \\
Other & $2(6.1)$ \\
Discharge diagnosis $(N=33), n(\%)$ & \\
\hline Pneumonia & $11(33.3)$ \\
Bronchiolitis & $1(3.0)$ \\
Upper airway obstruction & $2(6.1)$ \\
Sepsis & $9(27.3)$ \\
Meningitis & $4(12.1)$ \\
Other & $6(18.2)$
\end{tabular}

mortality on multiple regression analysis. There was no difference in gestational age or birthweight between infants who died v. those who survived (Table $1 ; p>0.4$ ).

\section{Discussion}

The present study is the first from sub-Saharan Africa investigating rehospitalisations of premature infants in any setting. Over the 6-month period, 29 infants were admitted to the PICU. Although premature infants in the first 6 months of life constituted only $5.7 \%$ of the total number of admissions in the study period, $11.6 \%$ of deaths in the unit were infants included in this study.

A higher PIM2 score on admission was associated with death during PICU admission ( $p=0.03$ ), as expected, ${ }^{[21]}$ and the SMR of 1.39 shows that the actual mortality was greater than the predicted mortality in this cohort. In 2016, the average PIM score for all PICU admissions was 5.68, and the average SMR was 1.1. This finding suggests that premature infants readmitted to the PICU may be a particularly vulnerable group with higher risk of mortality than their term counterparts; however, the observational nature of the study and lack of control group limit this conclusion, and further controlled studies are recommended to confirm this hypothesis.

The receipt of inotropes $(p<0.0001)$, a longer period of mechanical

Table 4. Cardiac arrests in PICU $(N=5)$

\begin{tabular}{lll}
\hline Gestational age (weeks) & $\begin{array}{l}\text { Birthweight } \\
\text { (grams) }\end{array}$ & $\begin{array}{l}\text { Reason for } \\
\text { admission }\end{array}$ \\
\hline 28 & 800 & Septic shock \\
28 & 1180 & Septic shock \\
32 & 1800 & $\begin{array}{l}\text { Apnoea } \\
34\end{array}$ \\
36 & 2490 & $\begin{array}{l}\text { Hypovolaemic } \\
\text { shock }\end{array}$ \\
& 2410 & Septic shock \\
PICU = paediatric intensive care unit. &
\end{tabular}

\section{Table 3. PICU course and outcomes $(N=29)$}

\begin{tabular}{|c|c|c|c|c|}
\hline Variable & All $(N=29), n(\%)^{*}$ & $\operatorname{Died}(n=5), n(\%)^{*}$ & Survived $(n=24), n(\%)^{\star}$ & $p$-value \\
\hline PIM2 score median (IQR) & $4.13(1.36-7.56)$ & $13.17(7.56-62.23)$ & $3.8(1.08-5.53)$ & 0.03 \\
\hline \multicolumn{5}{|l|}{ ICU, days } \\
\hline$<1$ & $2(6.9)$ & 0 & $2(8.3)$ & \multirow[t]{5}{*}{0.1} \\
\hline 1 & $3(10.3)$ & $2(40.0)$ & $1(4.2)$ & \\
\hline $2-3$ & $8(27.6)$ & $1(20.0)$ & $7(29.2)$ & \\
\hline $4-7 s$ & $8(27.6)$ & 0 & $8(33.3)$ & \\
\hline$>7$ & $8(27.6)$ & $2(40.0)$ & $6(25.0)$ & \\
\hline Mechanical ventilation & $21(72.4)$ & $5(100)$ & $16(66.7)$ & 0.1 \\
\hline \multicolumn{5}{|l|}{ Mechanical ventilation, days } \\
\hline None & $8(27.6)$ & 0 & $8(33.3)$ & \multirow[t]{6}{*}{0.03} \\
\hline$<1$ & $2(6.9)$ & 0 & $2(8.3)$ & \\
\hline 1 & $3(10.3)$ & $2(40.0)$ & $1(4.2)$ & \\
\hline $2-3$ & $3(10.3)$ & $1(20.0)$ & $2(8.3)$ & \\
\hline $4-7$ & $9(31)$ & 0 & $9(37.5)$ & \\
\hline$>7$ & $4(13.8)$ & $2(40.0)$ & $2(8.3)$ & \\
\hline Received inotropes & $6(20.7)$ & $5(100)$ & $1(4.2)$ & $<0.0001$ \\
\hline Blood transfusion & $14(48.3)$ & $4(80.0)$ & $1(4.2)$ & 0.1 \\
\hline Surgery $(n=7)$ or dialysis $(n=1)$ & $8(27.6)$ & $2(40.0)$ & $6(25.0)$ & 0.1 \\
\hline Cardiac arrest & $5(17.2)$ & $5(100)$ & 0 & $<0.0001$ \\
\hline
\end{tabular}


ventilation days $(p=0.03)$ and cardiac arrest in PICU $(p<0.0001)$ were all associated with mortality on univariate analysis. However, on multiple regression analysis, none of these factors was found to be an independent predictor of mortality.

No correlation was found between birthweight or gestational age at birth and mortality $(p \geq 0.4)$. Several studies have previously shown that both of these factors are associated with an increased risk of readmission. ${ }^{[5,9,13,14]}$ A lower gestational age is also associated with increasing risk of mortality. ${ }^{[22]}$ The majority of patients in the present study weighed $1500 \mathrm{~g}$ or more (median $1715 \mathrm{~g}$ ) and 58.6\% of these infants were moderate to late preterm. The small cohort of extremely and very preterm infants may explain why no correlation was found in this study.

Moderate to late preterm infants have been found to represent a large proportion of premature infants who require readmission and, as a result, utilise a significant amount of resources. ${ }^{[14]}$ This finding is reflected in the present study, but further research is required to truly quantify the morbidity and mortality associated with this group of infants. Interventions directed at this group of infants could potentially affect their health and progress dramatically after discharge from a neonatal unit.

Apnoea and respiratory failure were the primary reasons for PICU admission, as has been reported previously. ${ }^{[1,12,14,23]}$ Shock was another common reason for admission. On discharge, infections were the final diagnosis in $75.8 \%$ of admissions, including pneumonia, sepsis, meningitis and bronchiolitis, which is in keeping with a study from India. ${ }^{[18]}$ RSV infection was isolated in $3(9.1 \%)$ of the patients diagnosed with respiratory tract infection. Underwood et al. ${ }^{[14]}$ and Hong et al. ${ }^{[12]}$ both found that RSV-related illness was a significant cause of rehospitalisation. Obonyo et al. ${ }^{[24]}$ highlighted that there is a lack of harmonised criteria for sepsis with the paediatric community. The Surviving Sepsis Campaign International Guidelines for the Management of Septic Shock and Sepsis-associated Organ Dysfunction in Children ${ }^{[25]}$ published in February 2020 defined 'septic shock' as severe infection leading to cardiovascular dysfunction and 'sepsis-associated organ dysfunction' as severe infection leading to cardiovascular and/or noncardiovascular organ dysfunction. Our discharge diagnostic classifications aligned with this definition in that both infants with 'septic shock' and those with multi-organ dysfunction secondary to severe infection (pulmonary or non-pulmonary) were classified as having sepsis.

The median length of stay was previously found to be 3 days in this PICU. ${ }^{[26]}$ In this cohort, just over half (54.5\%) the admissions spent 4 or more days in PICU, with $\sim 20 \%$ of those patients having a PICU length of stay of 7 or more days. This finding suggests that these infants may utilise more resources when compared with the general population requiring admission to this PICU. Mourani et al. ${ }^{[7]}$ reported that in the first 4.5 years of life, $49 \%$ of the 96 premature infants admitted to PICU required mechanical ventilation for an average of 11.6 days. In our study, intubation and mechanical ventilation was required in a greater percentage (72.4\%) of admissions, but only 4 were ventilated for more than 7 days. Gunville et al. ${ }^{[17]}$ report that premature infants generally have a longer stay in PICU and utilise more hospital resources than infants born at term. Although the present study does not have the capacity to show similar findings, it does suggest that premature infants utilise a significant proportion of hospital resources, and further studies with term controls are warranted in this regard.

Infants with a lower gestational age at birth or with a lower BW were significantly more likely to require blood transfusion $(p \leq 0.05)$, which has previously been reported in infants weighing less than $1000 \mathrm{~g}$ at birth. ${ }^{[18]}$ It may be beneficial to routinely prescribe haematinics to all premature infants on discharge from the neonatal unit, as iron supplementation has been found to decrease the risk of developing iron deficiency anaemia in preterm infants. ${ }^{[27]}$

The majority of infants were male, in keeping with other studies. ${ }^{[5,9,12,28]}$ Seventeen (58.6\%) infants weighed $1500 \mathrm{~g}$ or more at birth and 17 (58.6\%) were born at or after 32 weeks of gestation. Although one study ${ }^{[10]}$ reported similar findings, most literature suggests that a lower GA or birthweight increases the risk of rehospitalisation. ${ }^{[5,13-15,29]}$ Our findings suggest that moderate to late preterm infants are also susceptible to the consequences of prematurity in this setting and should therefore receive the same care and follow-up as extremely preterm infants.

Thirty-eight percent of infants in our study were missing immunisations, which is concerning in terms of public health and preventive health care. Although B. pertussis infection was presumed in 1 case, the affected infant had received 3 doses of the vaccine for $B$. pertussis. No other EPI-administered vaccine-preventable causes of admission were identified. RSV prophylaxis (palivizumab) is not routinely available in the study region, despite being recommended during the RSV season for children born at a gestational age $<36$ weeks, who are younger than 6 months. ${ }^{[30]}$ Only 1 of the 3 children affected by RSV-related respiratory illnesses would have fulfilled the criteria to receive RSV prophylaxis, if it were available. Therefore 1 of the $33(3.0 \%)$ admissions was potentially avoidable had palivizumab been available in the neonatal units during the study period.

It was fairly reassuring to find that all the infants were HIVuninfected on admission, including those at risk for mother-tochild transmission, and the vast majority were receiving exclusive breastmilk feeds. Despite this, only $48.3 \%$ of the infants were noted to be growing well and $31 \%$ of the infants had either lost weight since discharge from the neonatal unit or were categorised as malnourished. Most of the infants were admitted more than a month after discharge from the neonatal unit. Early identification and timeous intervention in infants who are not growing well is required to optimise the health and well-being of all premature infants.

The present study is the first to explore readmissions of ex-premature infants into a PICU in South Africa. The population of severely unwell patients whom this centre cares for generally live in poor socioeconomic circumstances and many present late in the course of illness, owing to challenges in accessing health services. ${ }^{[31]}$ Patients generally have limited access to private transport and the duration of the pathway to PICU has been found to be unacceptably long. ${ }^{[31]}$ In this resource-limited setting, the additional morbidity associated with prematurity may have significant implications for the patient, family and healthcare service. Studies of this particularly vulnerable patient group are therefore important to identify and implement preventive strategies as well as targeted interventions to optimise care of premature and ex-premature infants.

Limited research has previously shown that programmes that improve parental education may lead to a decreased rate of readmission in infants who are discharged from a neonatal unit. ${ }^{[32]}$ This aspect could potentially improve the overall health of this vulnerable population if parents are actively involved in the care of their child, particularly regarding immunisations, breastfeeding and monitoring of growth. If caregivers are empowered to identify signs of illness in their infant, they may present earlier in the course of illness.

Improved access to antenatal care and family planning services to decrease the incidence of prematurity must become, and remain, a priority for all those involved in the provision of healthcare. Health promotion regarding breastfeeding support, regular visits to primary 
healthcare facilities for monitoring of growth and wellness, as well as promoting immunisations provided through the EPI programme needs to become a major focus of the care of all infants and, in particular, those born preterm.

\section{Study limitations}

This study has several limitations. The lack of a control group increases the likelihood of confounding bias within the study. The small study sample as well as the short study period affect the validity of the study findings. The small study sample may reflect data collection during a period of low respiratory infection prevalence. The information related to neonatal admission was mostly found in the Road to Health booklet, and some details regarding neonatal admission may have been incomplete, resulting in potential information bias. This study did not include any infants who may have been rehospitalised in a PICU in another hospital or other districts in South Africa.

\section{Conclusion and recommendations}

Over a 6 -month period, $5.7 \%$ of the total admissions and $11.6 \%$ of the deaths in PICU were of premature infants within the first 6 months of life, with a $S M R>1$. Independent predictors of mortality could not be identified in this small cohort. The study suggests that premature infants utilise a considerable proportion of PICU resources and are at increased risk of serious illness and mortality. Moderate to late preterm infants may be a particularly vulnerable group, and more research into this cohort of infants is essential. Further research is required to clarify the extent of resource utilisation, morbidity and overall mortality in this population of infants.

Prematurity remains a significant risk factor for morbidity, and further studies are required to gain insight into the true burden of disease associated with prematurity in Southern Africa, including the healthcare resource consumption outside the PICU. Interventions directed at improving nutritional status and promoting the use of immunisations should be focused on, as this remains a problem in this cohort of patients. Parental education and empowerment may have a significant impact in this regard. Preventive healthcare is a priority, specifically in terms of decreasing the incidence of premature deliveries as well as improving healthcare for all mothers, infants and children.

\section{Declaration. None.}

Acknowledgements. None.

Author contributions. GM collected the data and drafted the manuscript; BM analysed the data; all authors contributed to study protocol development, interpretation of results and writing the manuscript. Funding. None.

Conflicts of interest. None.

1. World Health Organization. Children: Reducing mortality. Weekly Epi Record 2014;89(38):418-428. https:/www.who.int/wer/2014/wer8938/en/ (accessed 17 January 2017)

2. Reid AE, Hendricks MK, Groenewald P, et al. Where do children die and what are the causes? Under- 5 deaths in the Metro West geographical service area of the Western Cape, South Africa, 2011. S Afr Med J 2016;106(4):359-364. https://doi.org/10.7196/samj.2016.v106i4.10521

3. Vidyasagar D. Half a century of evolution of neonatology: A witness's story. Ind J Pediatr 2015;82(12):1117. https://doi.org/10.1007/s12098-015-1838-8

4. Shah PS. Neonatal intensive care - the only constant is change. N Engl J Med 2017;376(7):694-696. https://doi.org/10.1097/01.aoa.0000521248.25115.9a

5. Tseng YH, Chen CW, Huang HL, et al. Incidence of and predictors for short-term readmission among preterm low-birthweight infants. Pediatr Int 2010;52(5):711-717. https://doi.org/10.1111/j.1442-200x.2010.03129.x
6. Slimings C, Einarsdottir K, Srinivasjois R, et al. Hospital admissions and gestational age at birth: 18 years of follow up in Western Australia. Paediatr Perinat Epidemiol 2014;28(6):536-544. https://doi.org/10.1111/ppe.12155

7. Mourani PM, Kinsella JP, Clermont G, et al. Intensive care unit readmission during childhood after preterm birth with respiratory failure. J Pediatr 2014;164(4):749-755.e3. https://doi.org/10.1016/j.jpeds.2013.11.062

8. Elder DE, Hagan R, Evans SF, et al. Hospital admissions in the first year of life in very preterm infants. J Paediatr Child Health 1999;35(2):145-150. https://doi. org/10.1046/j.1440-1754.1999.00308.x

9. Smith VC, Zupancic JA, McCormick MC, et al. Rehospitalisation in the first year of life among infants with bronchopulmonary dysplasia. J Pediatr 2004;144(6):799-803. https://doi.org/10.1016/j.jpeds.2004.03.026

10. Escobar GJ, Joffe S, Gardner MN, et al. Rehospitalisation in the first two weeks after discharge from the neonatal intensive care unit. Pediatrics 1999;104(1):e2. https://doi.org/10.1542/peds.104.1.e2

11. Ralser E, Mueller W, Haberland C, et al. Rehospitalisation in the first 2 years of life in children born preterm. Acta Paediatr 2012;101(1):e1-e5. https://doi. org/10.1111/j.1651-2227.2011.02404.x

12. Hong $T$, Bolisetty $S$, Bajuk $B$, et al. A population study of respiratory rehospitalisation in very preterm infants in the first 3 years of life. J Paediatr Child Health 2016;52(7):715-721. https://doi.org/10.1111/jpc.13205

13. Brissaud O, Babre F, Pedespan L, et al. Rehospitalisation of very preterm infants in the first year of life. Comparison of 2 groups: 1997 and 2002. Arch Pediatr 2005;12(10):1462-1470. https://doi.org/10.1016/j.arcped.2005.04.078

14. Underwood MA, Danielsen B, Gilbert WM. Cost, causes and rates of rehospitalisation of preterm infants. J Perinatol 2007;27(10):614-619. https://doi. org/10.1038/sj.jp.7211801

15. Kuint J, Lerner-Geva L, Chodick G, et al. Rehospitalization through childhood and adolescence: Association with neonatal morbidities in infants of very low birth weight. J Pediatr 2017;188:135-141.e2. https://doi.org/10.1016/j. jpeds.2017.05.078

16. Taylor JB, Go MA, Nyp MF, et al. Rehospitalisation in infants born $<29$ weeks' gestation during the first 2 years of life: Risk assessment. Am J Perinatol 2016;33(1):24-33. https://doi.org/10.1055/s-0035-1555123

17. Gunville CF, Stratton KA, Sontag MK, et al. The scope and impact of early and late preterm infants admitted to the PICU with respiratory illness. J Pediatr 2010;157(2):209-214.e1. https://doi.org/10.1164/ajrccm-conference.2009.179.1_ meetingabstracts.a5979

18. Mukhopadhyay K, Louis D, Mahajan G, et al. Longitudinal growth and postdischarge mortality and morbidity among extremely low birth weight neonates. Indian Pediatr 2014;51(9):723-726. https://doi.org/10.1007/s13312-014-0489-6

19. Blencowe H, Cousens S, Chou D, et al. Born too soon: The global epidemiology of 15 million preterm births. Reprod Health 2013;10(Suppl 1):S2. https://doi. org/10.1186/1742-4755-10-s1-s2

20. Slater A, Shann F, Pearson G. PIM2: A revised version of the Paediatric Index of Mortality. Intensive Care Med 2003;29(2):278-285. https://doi.org/10.1007/ s00134-002-1601-2

21. Solomon LJ, Morrow BM, Argent AC. Paediatric index of mortality scores: An evaluation of function in the paediatric intensive care unit of the Red Cross War Memorial Children's Hospital. S Afr J Crit Care (online) 2014;30:8-13. https:// doi.org/10.7196/sajcc.166

22. Stephens AS, Lain SJ, Roberts CL, et al. Survival, hospitalisation, and acutecare costs of very and moderate preterm infants in the first 6 years of life: A population-based study. J Pediatr2016;169:61-68.e3. https://doi.org/10.1016/j. jpeds.2015.10.028

23. Ambalavanan N, Carlo WA, McDonald SA, et al. Identification of extremely premature infants at high risk of rehospitalisation. Pediatrics 2011;128(5):e1216-e1225.

24. Obonyo NG, Schlapbach LJ, Fraser JF. Sepsis: Changing definitions, unchanging treatment. Front Pediatr 2019;6:425. https://doi.org/10.3389/fped.2018.00425

25. Weiss SL, Peters MJ, Alhazzani W. Executive summary: Surviving sepsis campaign international guidelines for the management of septic shock and sepsisassociated organ dysfunction in children. Pediatr Crit Care Med 2020;21(2):186195. https://doi.org/10.1097/PCC.000000000002197

26. Nupen TL, Argent AC, Morrow BM. Characteristics and outcomes in long-stay patients in a paediatric intensive care unit in Cape Town, South Africa. S Afr Med J 2016;107(1):70-75. https://doi:10.7196/SAMJ.2017.v107i1.11279

27. Mills RJ, Davies MW. Enteral iron supplementation in preterm and low birth weight infants. Cochrane Database Syst Rev 2012;(3):CD005095. https://doi. org/10.1002/14651858.cd005095.pub2

28. Escobar GJ, McCormick MC, Zupancic JA, et al. Unstudied infants: Outcomes of moderately premature infants in the neonatal intensive care unit. Arch Dis Child Fetal Neonatal Ed 2006;91(4):F238-F244. https://doi.org/10.1136/ adc. 2005.087031

29. Lee JH, Chang YS, Committee on Data Collection and Statistical Analysis, the Korean Society of Neonatology. Use of medical resources by preterm infants born at less than 33 weeks' gestation following discharge from the neonatal intensive care unit in Korea. J Korean Med Sci 2015;30 Suppl 1:S95 -S103. https://doi.org/10.3346/jkms.2015.30.s1.s95 
30. Zar HJ, Madhi SA, White DA, et al. Acute viral bronchiolitis in South Africa: Strategies for management and prevention. S Afr Med J 2016;106(4):330-332. https://doi.org/10.7196/samj.2016.v106i4.10437

31. Hodkinson P, Argent A, Wallis L, et al. Pathways to care for critically ill or injured children: A cohort study from first presentation to healthcare services through to admission to intensive care or death. PLoS ONE 2016;11(1):e0145473. https://doi.org/10.1371/journal.pone.0145473
32. Bastani F, Abadi TA, Haghani H. Effect of family-centered care on improving parental satisfaction and reducing readmission among premature infants: A randomised controlled trial. J Clin Diagn Res 2015;9(1):SC04-SC8. https:// doi.org/10.7860/JCDR/2015/10356.5444

Accepted 20 July 2020 